\title{
Autophagy Modulation As a Potential Therapeutic Target for Liver Diseases
}

\author{
Pankaj Puri*, Alok Chandra \\ ${ }^{*}$ Department of Gastroenterology, Army Hospital (Research and Referral), Dhaula Kuan, New Delhi 110010, and 'Department of \\ Gastroenterology, Command Hospital (EC), Alipore Road, Kolkata 700027, West Bengal, India
}

\begin{abstract}
Autophagy is a critical intracellular pathway which maintains cellular function by lysosomal degradation of damaged proteins and organelles besides elimination of invading pathogens. Its primary function is to prevent cell death. Autophagy has diverse physiological functions namely; starvation adaptation, prevention of tumorigenesis, energy homeostasis, intracellular quality control and degradation of abnormal intracellular protein aggregates. Understanding the molecular mechanisms of autophagy has given key insights into the pathogenesis of various diseases like Non Alcoholic Steato-Hepatitis, Hepatitis B and C infections, Alpha-1 antitrypsin deficiency and hepatocellular carcinoma. Pharmacological modulation of autophagy may have a therapeutic potential in management of these liver diseases. (
\end{abstract}

$\mathrm{O}$ urobros or Urobros is an ancient symbol depicting a serpent or a dragon eating its own tail, which symbolizes cyclicality in the sense of something recreating itself. In living organisms, cells and intracellular organelles need to be constantly remodeled and renewed. Autophagy is a term derived from Greek terminology for self-eating and signifies the process of cellular selfdigestion in which cytoplasmic components are degraded in lysosomes. Reminiscent of the serpent eating its own tail in the symbol of Ourobros, the cells are able to survive by using autophagy to remove damaged proteins and organelles, eliminate invading microbes and generate nutrients during starvation.

After Metchnicoff, a Russian zoologist, first described phagocytosis, the role of lysosomes and autophagy in degrading cytoplasmic components has been elucidated. ${ }^{1,2}$ The control of autophagy has been recently delineated by identification of over 30 Autophagy-related (ATG) genes. $^{3}$

Autophagy functions to prevent rather than promote cell death. Autophagy has effects of increased cytoprotection, decreased stem cell attrition, decreased oncogenic

Keywords: autophagy, autophagosome, liver diseases

Received: 10.2.2014; Accepted: 1.4.2014; Available online: 18.4.2014

Address for correspondence: Pankaj Puri, Department of Gastroenterology, Army Hospital (Research and Referral), Dhaula Kuan,

New Delhi 110010, India.

E-mail: puripankaj@gmail.com

Abbreviations: ULK1: Uncoordinated 51-like kinase 1; mTORC1: mTOR complex 1; ER: endoplasmic reticulum; mTORC2: mTOR complex 2; NASH: Non Alcoholic Steato-Hepatitis; TG: triglyceride; HSC: hepatic stellate cells; STEBPs: sterol regulatory element-binding proteins; AMPk: adenosine monophosphate-activated protein linase; AT: antitrypsin; HBV: hepatitis B virus; HCV: hepatitis C virus; HCC: hepatocellular

carcinoma

http://dx.doi.org/10.1016/j.jceh.2014.04.001 transformation, decreased dysfunctional mitochondria and decrease in dysfunctional aggregate prone proteins. ${ }^{4}$ The role of mammalian target of Rapamycin (mTOR) complex in the process of autophagy has been elucidated and induction of autophagy by mTOR inhibition provided an insight into the prospect for its modulation in health and disease. ${ }^{5,6}$

\section{MECHANISM OF AUTOPHAGY}

In its simplest form, as in yeast, it represents the cells adaptation to starvation. However, if autophagy is unregulated, it can be harmful. Hence, it needs to be tightly regulated to maintain cell homeostasis.

Three types of autophagy are described, namely, macroautophagy, chaperone mediated autophagy and microautophagy. In microautophagy, there is direct uptake of cytoplasm or organelles at lysosomal surface by inavgination and protrusion of lysosomal membrane. Chaperone mediated autophagy targets only a subset of proteins that have a specific peptide motif, KFERQ and is mediated by molecular chaperones like heat shock protein 70 which recognize and bind to the peptide motif of the substrate protein. ${ }^{7,8}$ In macroautophagy (hereinafter referred to as autophagy), isolated membranes (phagophores) engulf a portion of the cytoplasm and some organelles resulting in the formation of vacuoles surrounded by a double membrane called autophagosomes. The phagosome further fuses with the lysosome to form the "autolysosome". Degradation of material within follows after fusion.

Multiple Atg proteins regulate autophagosome formation. Multiple Atg proteins have been identified, of which, Atg 1-10, 12-14, 16 and 18 are called "core proteins" as they are essential for autophagosome formation. ${ }^{9}$ These core proteins along with other proteins have been divided 
into four subgroups referred to as "core molecular mechanisms". They are (i) Uncoordinated 51-like kinase 1 (ULK1) complex which controls induction of autophagy and is negatively regulated by mTOR; (ii) Beclin 1-class III P13K complex which controls nucleation of the autophagosome; (iii) Two ubiquitin-like conjugation systems (Atg12-Atg5 system and LC3 system) which mediate the elongation stage and; (iv) Atg9 retrieval process which involves Atg18-Atg2 complex. Atg9 is postulated to contribute to delivery of membranes required in autophagosome formation. $^{10-18}$

The core machinery of autophagy comprises of four steps. These are:

a) Induction: the process of autophagosome formation starts with the dissociation of mTOR complex 1 (mTORC1) from ULK1 complex. Under nutrient rich conditions, mTORC1 is attached with the ULK1 complex. Autophagy is initiated during conditions of high energy demand as in starvation when the mTORC1 dissociates from the ULK1 complex.

b) Vesicle nucleation: during this step recruitment of proteins and lipids for autophagosome formation occurs. Beclin 1-class III P13K complex controls nucleation of the autophagosome.

c) Vesicle expansion and completion: membranes assemble at PAS directly from ER or by de-novo vesicular addition and then seal to form the autophagosome. Two ubiquitin-like conjugation systems (Atg12-Atg5 system and LC3 system) mediate the elongation stage.

d) Autolysosome formation: the outer layer of the autophagosomes fuses with the lysosomes (to form an auto- lysosome) resulting in degradation of the sequestered material. ${ }^{19,20}$

The autophagy pathway and the core autophagic machinery are depicted in Figure 1.

\section{PHYSIOLOGICAL ROLE OF AUTOPHAGY}

\section{Autophagy and Apoptosis}

Autophagy and apoptosis are important cellular functions required for mitigation of cellular stress and help in prevention of disease. Mostly, autophagy and apoptosis are independent processes. However, autophagy may also modulate apoptosis in certain conditions of cellular stress. Many cellular regulators of autophagy and apoptosis, like Bcl-2 family, Atg5, Beclin1 and Atg4D are also common. ${ }^{21}$ Degradation of caspase- 8 by autophagy inhibits cell death by apoptosis, thus contributing to the role of autophagy in regulation of apoptosis. ${ }^{22}$ Several Atg proteins have a role in autophagy-independent apoptosis. Also, various apoptosis-inducing factors may also have a role in regulation of autophagy. This cross-talk between autophagy, which is primarily a cell-survival mechanism, and apoptosis, which is primarily a cell-death mechanism, is complex. An understanding of these mechanisms and its regulation may form the framework for using autophagy modulation for therapeutic purpose in future. ${ }^{23}$

\section{Starvation Adaptation}

Autophagy also plays an important role in situations of prolonged fasting. It ensures glucose supply prior to onset

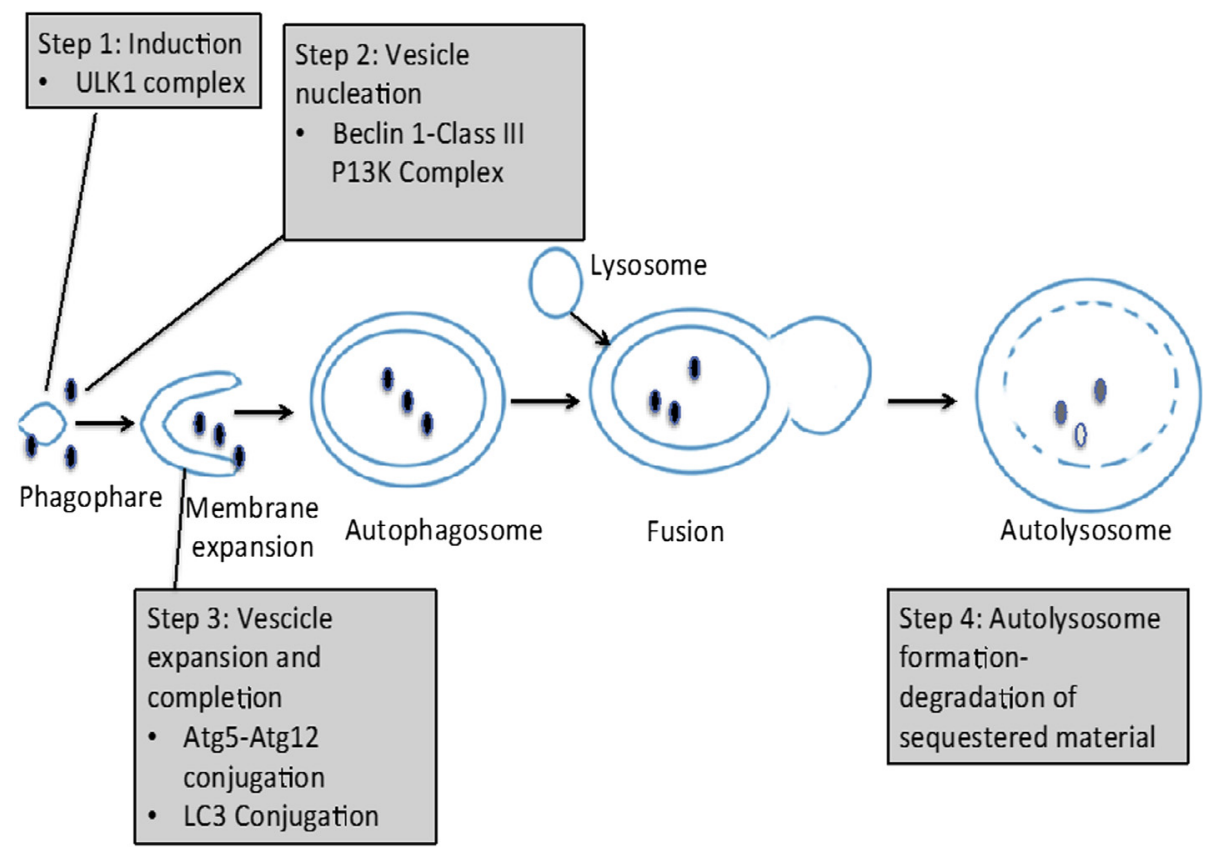

Figure 1 The autophagy pathway and the core autophagic machinery. 
of gluconeogenesis by creation of gluconeogenic amino acids. Thirty to forty percent of liver proteins are catabolized within $48 \mathrm{~h}$ of fasting to provide the body with amino acids. ${ }^{24}$ Experimental Atg7 deficient mice do not exhibit either the decrease in liver proteins or the increase in amino acids on fasting. ${ }^{25}$ Free fatty acids are required for energy production by $\beta$ oxidation during periods of starvation. Autophagy supplies it by releasing free fatty acids from liver and accumulating it in various cells as lipid droplets in combination with triglycerides. These lipid droplets are again broken down to provide the substrate for $\beta$ oxidation. $^{26}$

\section{Tissue Homeostasis and Renovation}

Basal levels of autophagy help in recycling tissue components and organelles to prevent accumulation of damaged products in cells which may result in cell death or disease. Approximately $1-5 \%$ of cellular proteins are recycled in the liver by autophagy every hour. ${ }^{27}$ Atg7 deficient mice show swollen and deformed mitochondria in liver cells and appearance of concentric membranous structures comprised of endoplasmic reticulum (ER). They also show increased number of lipid droplets, peroxisomes and protein aggregates. This can lead to severe hepatomegaly and hepatitis. ${ }^{28}$ The removal of harmful cellular aggregates by inducing autophagy has been tested in experimental models of $\alpha 1$ antitrypsin deficiency. ${ }^{29}$

\section{Prevention of Tumorigenesis}

Although cancer is primarily due to unregulated cell division, its prevention by autophagy, which is primarily a process for cell survival, may seem paradoxical. Metabolic stress usually leads to cell death by apoptosis. If the cell death by apoptosis does not occur, necrosis takes over for clearing damaged cells. This leads to increased tissue inflammation and may increase tumor formation and growth. Autophagy ameliorates this process by clearing the cells not cleared by apoptosis and preventing necrosis. It also prevents disordered cell growth during periods of metabolic stress, thus preventing tumorigenesis. ${ }^{30,31}$

The mTOR is an important signaling pathway for maintaining cell homeostasis. It interacts with a variety of environmental signals to affects various cellular processes. It aggregates with various proteins to form two distinct complexes called mTOR complex 1 (mTORC1) and mTOR complex 2 (mTORC2). ${ }^{5}$ Loss of p53, a common tumor suppressor gene, is common to many cancers. This leads to mTORC1 activation as the upstream pathway in pathogenesis. ${ }^{32}$ It has been demonstrated that constant activation of mTORC1 leads to reduced autophagy and may contribute to increased tumorigenesis. In experimental models, knockout models of various proteins required for autophagy demonstrated increased cancers, including spontaneous hepatocellular carcinoma. $^{32-35}$

\section{Aging and Longevity}

Autophagy is primarily a cytoprotective process. It mediates this process in various organ systems and can protect against the effects of aging. Inhibition of Insulin like growth factor pathway in Caenorbabditis elegans increases autophagy and may contribute to longevity. ${ }^{36-39}$ Calorie restriction is a key anti-aging intervention in most animals. It has also been found to be a physiological inducer of autophagy, thus further underlining the role of autophagy in prolonging longevity. ${ }^{31,40}$ Autophagy balances the excess necrotic or apoptotic cell death in actively dividing cells. This is possibly related to release of Bcl-2 from Beclin1 thus blocking apoptosis. ${ }^{41}$ Many oncogenes modulate their effects by suppression of autophagy. Also, by improving the cell quality control, autophagy can suppress de-novo oncogenesis. ${ }^{42,43}$ It also improves innate immunity and decreases the inflammatory response, thus exerting anti-aging affect independent of its effect on cells. $^{44}$

Increased liver injury is seen in aged mice compared to younger mice. This increased injury is likely to be due to increased apoptosis as evidenced by increased caspase- 3 activity. ${ }^{45}$ Besides the beneficial effects of calorie restriction on the aging process, resveratrol has also been shown to be effective in experimental models. ${ }^{4}$ However further studies are required to see whether resveratrol or other pharmacological measures to increase autophagy result in improved liver functions.

\section{Autophagy in Maintenance of Homeostasis in Liver and Hepatic Energy Metabolism}

Basal rates of autophagy are essential in maintaining hepatocyte homeostasis. It acts mainly by clearing misfolded proteins, protein aggregates and removing damaged mitochondria. ${ }^{20}$ This is amply demonstrated in $\alpha 1$ Antitrypsin deficiency where accumulation of mutant proteins in the ER form intra-hepatic inclusions.

Experimental Atg7 deficient mouse livers show accumulation of misfolded proteins, deformed mitochondria and lipid droplets. It leads to marked hepatomegaly, hepatitis and de-novo tuomurigenesis. ${ }^{28}$

Selective degradation of intracellular organelles like the mitochondria, ER and the Golgi apparatus are also important functions of basal autophagy to maintain normal turnover and function of these organelles. They are also referred to as mito-phagy and ER-phagy. Any disruption can lead to damaged and dysfunctional organelles. ${ }^{46}$

Starvation is the most important inducer of autophagy. It plays a crucial role in protein catabolism in the liver. In wild type mice, $25-40 \%$ of liver protein is mobilized as amino acids within $48 \mathrm{~h}$ of starvation. It is mainly derived from cytosolic proteins. ${ }^{47}$ Atg7 deficient mice do not exhibit these levels of protein loss and consequent amino acid levels increase. They also demonstrate hypoglycemia 
in contrast to wild type mice, suggesting a role for autophagy in gluconeogenesis. ${ }^{48}$

Autophagy is an ATP dependent process. During periods of nutrient deficiency, autophagy is promoted by AMPK by inhibiting suppressive effect of mTORC1 or directly via activating ULK1. ${ }^{49}$ In the newborn also, provision of glucose by glycogen degradation is an important role of autophagy until provision of nutrients by milk takes place. ${ }^{50}$

\section{AUTOPHAGY MODULATION IN THERAPY FOR LIVER DISEASES}

\section{Targeting Autophagy in Non Alcoholic Steato- Hepatitis (NASH)}

Excessive liver lipid deposition in form of triglycerides stored in vesicles and abnormal lipid metabolism characterize NASH. Liver injury progresses by effect of oxidant stress and cytokines as cofactors.

Similar to the degradation of cytoplasmic proteins and organelles, autophagy has also been found to be involved in selective degradation of these lipid droplets. This has also been termed as Lipophagy. ${ }^{51}$ Liver triglyceride (TG) content is increased in Atg7 deficient mice, suggesting an important role of autophagy in its regulation. ${ }^{52}$ Inhibition of autophagy by pharmacological inhibition of Atg5 in cultured hepatocytes show an abnormal increase in cellular TG content on challenge with a lipid load. Experimental mice with obesity and consequently increased hepatocyte lipid content demonstrated a decrease in indicators of autophagy. Restoration of Atg7 expression in these obese mice has been shown to be beneficial by reduction in ER stress, decreased serum insulin levels, improved glucose tolerance and decreased liver TG content. ${ }^{53}$

Insulin resistance is strongly associated with development of NASH. Decreased levels of basal autophagy have also been linked to hyperinsulinemia and insulin resistance. High fatty diet fed mice showed decreased levels of autophagy in liver evidenced by decreased levels of p62, a protein degraded by macro-autophagy, and decreased levels of Atg5 and Atg7..$^{54}$

NASH is also characterized by hepatic inflammation and fibrosis. This is partly mediated by oxidative stress and cytokines. Autophagy regulates TNF and oxidative stress associated cell death and may modulate progression of liver disease in NASH as well. ${ }^{55}$ Damaged mitochondria have been found to accumulate in the hepatocytes in NASH and a possible defect in mitophagy may contribute to progression of liver damage. ${ }^{56}$

Autophagy has also been postulated to be involved in activation of hepatic stellate cells (HSC) with consequent deposition of extracellular matrix and liver fibrosis. The postulated mechanisms include its effect on HSC fat stores in from of vitamin A or a direct effect on its differentiation. ${ }^{57}$
The mTOR complex is involved with regulating autophagy and is also essential for the regulation of lipid biosynthesis. The mTOR suppression has two beneficial effects in NASH. Firstly, it induces autophagy resulting in lipophagy. Secondly, mTOR positively regulates sterol regulatory element-binding proteins (STEBPs). Suppression of mTOR results in suppression of DREBPs mediated lipogenesis. Suppression of mTOR by TORIN-1 causes dephosphorylation of Lipin 1, which promotes its nuclear localization. This results in decreased SREBP-1-mediated lipogenesis by reducing the abundance of nuclear SREBP-1 protein. $^{58}$

Since there is no definitive pharmacological treatment of NASH, development of an effective treatment by targeting autophagy would be a significant development. Increase in liver autophagy can be used as a potential therapeutic target in treatment of NASH. The potential beneficial effects are:

i) Decreased triglyceride and cholesterol levels in hepatocytes

ii) Improved insulin homeostasis

iii) Decreased cellular oxidative stress

iv) Decreased TNF mediated liver injury

v) Better turnover of damaged ER and mitochondria in hepatocytes

vi) Decreased chances of malignancy. ${ }^{59}$

\section{Targeting Autophagy in Alcoholic Liver Disease}

Excess alcohol consumption can lead to a spectrum of liver injuries including steatosis, fibrosis and development of cirrhosis. There are various direct and indirect evidences to suggest that autophagy is suppressed in alcoholic liver disease. There is increased protein accumulation and decreased clearance from the hepatocytes. ${ }^{60}$ MalloryDenk bodies are intracellular inclusions found in alcoholic liver disease. These are protein aggregates and autophagy participates in its elimination. In experimental mice with loss of autophagy functions, similar inclusions are formed in the hepatocytes. ${ }^{61}$ Ethanol feeding diminishes the proteolytic capacity of liver lysosomes. ${ }^{62}$ The postulated mechanism of decreased autophagy levels in alcohol abuse may be due to decreased adenosine monophosphate-activated protein linase (AMPk) activity with consequent decrease in autophagy via the mTOR pathway and altered intracellular vesicle transport due to altered functions of microtubules and microfilaments. ${ }^{63}$ The mTOR inhibitors may be beneficial in alcoholic liver disease by inducing apoptosis, improving the clearance of proteins from the hepatocytes as well as suppressing lipogenesis.

\section{Targeting Autophagy in Alfa-1 Antitrypsin Deficiency}

This is a genetic disease characterized by accumulation of a mutant protein within the endoplasmic reticulum of 
the hepatocytes. The alfa- 1 antitrypsin (AT) protein is synthesized in hepatocytes and secreted into the blood where it acts as an inhibitor for neutrophil proteases. A mutation in the AT gene results in accumulation of the AT protein in the endoplasmic reticulum. This mutant protein cannot be degraded by the proteasome, which is the normal pathway for degradation of wild type protein. Autophagy forms an important pathway for mobilization of misfolded mutant protein aggregates. ${ }^{64}$ Although the exact mechanism of liver injury in alpha 1 AT deficiency is unknown, increase in autophagy induction by carbamazepine has been tested to mobilize the protein aggregates and decrease hepatic fibrosis. Carbamazepine has been shown to reduce these inclusions in mouse models by induction of autophagy along with reduced liver injury and fibrosis. ${ }^{29}$ Carbamazepine induces autophagy through a mTOR independent pathway as Rapamaycin has no effect on mutant AT protein aggregate degradation. There are other mTOR independent autophagy inducers including lithium, sodium valproate, verapamil, loperamide, amiodarone, nimodipine and nitrendipine but their role in AT-deficiency induced liver injury needs to be defined. ${ }^{51}$

A rare inherited disease, fibrinogen storage disease, is characterised by hypofibrinogenemia and accumulation of fibrinogen aggregates in the hepatocytes. This is similar to alpha 1 AT deficiency and stimulation of autophagy by carbamazepine has been shown to help in clearing abnormal protein aggregates, prevent further deposition of abnormal protein and retard fibrosis progression. ${ }^{65}$

\section{Targeting Autophagy in Infective Diseases: Hepatitis B virus (HBV) and Hepatitis $C$ virus (HCV) infection}

Autophagy is emerging as an important component in the antimicrobial defense against diverse infections. However, the microbes have also evolved mechanism to evade and exploit autophagy and both HBV and HCV have adapted to use autophagy for their own survival. ${ }^{66}$

$\mathrm{HCV}$ utilizes various autophagy components for its own replication. ${ }^{67}$ Studies have shown that there is an accumulation of autophagic vacuoles in HCV-infected hepatocytes; however this autophagy is inefficient as the autophagosomes are not able to increase autophagic protein degradation. The strategies used by HCV to evade and take advantage of autophagy for its replication are: (i) $\mathrm{HCV}$ avoids recognition by the autophagic process with rare or no co-localization of HCV proteins in autophagic vacuoles $^{68-70}$; (ii) HCV prevents maturation of autophagosomes into autolysosomes ${ }^{68,69}$; and (iii) HCV utilizes components of autophagy to enhance intracellular replication. The autophagy proteins are likely to be proviral factors for the initial steps of HCV replication. ${ }^{67-69}$

The autophagy machinery is also subverted in HBV. The autophagosomes are also induced in HBV; but, unlike
$\mathrm{HCV}$, the late autophagic vacuoles are detectable in hepatocytes. However, there is increase in protein degradation in HBV infected cells. ${ }^{71}$ In HBV infection, the HBVencoding protein, $\mathrm{HBx}$, plays a crucial role in subverting the autophagy functions in infected cells. Autophagy enhances viral replication at most steps of HBV replication. ${ }^{72}$

Since autophagy promotes the replication of both HBV and $\mathrm{HCV}$, it is possible that pharmacological suppression of autophagy may suppress viral replication.

\section{Targeting Autophagy in Liver Fibrosis}

Hepatic fibrosis is the final outcome of liver injury due to diverse etiologies and can progress to cirrhosis and hepatocellular carcinoma (HCC). Liver fibrosis is caused by activated myofibroblasts which may be derived from activation of quiescent hepatic stellate cells; bone marrowderived fibrocytes or circulating mesenchymal cells; or transition of other cells like hepatocytes, cholangiocytes and endothelial cells to mesenchymal cells to become myofibroblasts. Activated hepatic stellate cells produce extracellular matrix and collagen resulting in fibrosis and scarring.

Induction of autophagy in hepatic stellate cell may promote its activation and proliferation. ${ }^{73}$ However, there is conflicting data on the role of autophagy in hepatic fibrosis and further studies are required before autophagy can be targeted as a therapeutic approach to treat hepatic fibrosis. ${ }^{49}$

\section{Targeting Autophagy in Hepatocellular Carcinoma (HCC)}

The role of autophagy in HCC has been relatively well documented. It is mainly a tumor suppressor process. The possible mechanisms have been demonstrated in various in-vitro, animal and human studies. Beclin 1 forms a complex which promotes membrane nucleation during autophagy. While homozygous Beclin1 knockout mice do not survive, heterozygous Beclin1 knockout mice survive. An increased frequency of spontaneous tumors including liver tumors have been detected in these heterozygous Beclin1 knockout mice. ${ }^{74}$ The tumor-suppressing role of autophagy in HCC has been shown by demonstrating that most aggressive malignant HCC cell lines and HCC tissues with recurrent disease display much lower autophagic levels than less aggressive cell lines or tissues. HCC cell lines have consistently demonstrated a decreased expression of several genes required for autophagy like ATG5, ATG7 and BECLIN1. Their expression was however normal in adjacent tissue from same patient, thus prompting to their role in carcinogenesis. The expression of Beclin1 has also been correlated with disease free survival after curative resection. ${ }^{75}$ Rapamycin a known inducer of autophagy has been associated with better survival in patients with HCC who underwent liver transplantation. 
Autophagy shows a "double face" in HCC. While autophagy helps to prevent tumorigenesis, it is also used by the cancer cells for survival against apoptosis by traditional chemotherapeutic drugs. Its inhibition may also restore the cancer cell sensitivity to chemotherapy. ${ }^{76}$

Metastasis is a major cause of death due to hepatocellular carcinoma. Tumor cells may use autophagy to survive in a poor nutrient environment and this may promote colonization in distant organs. Experimental studies have confirmed the upregulation of autophagy in HCC metastasis. Modulation of autophagy may have a role in preventing tumor metastasis and survival. ${ }^{77}$

The modulation of autophagy has been proposed as a novel therapeutic target for therapy of HCC. ${ }^{78}$ However, since autophagy has a role in both cell survival and cell death in HCC under different conditions, both autophagy inducers as well as inhibitors have been tried as novel therapeutic strategies. A variety of cancer therapeutic agents have been used with inducers of autophagy for possible enhanced cell death. Various agents used for inducing autophagy are Rapamycin and its analogs, Tyrosine kinase inhibitors like sorafenib, NPC-16 a novel agent and cannabinoids and its agonists. ${ }^{79-81}$

Autophagy inhibitors have also been tried for their possible role in potentiating the effect of chemotherapeutic agents in treatment of cancer by abolishing the protective effect of autophagy on cell survival. Chloroquine and Hydroxychloroquine have been used as autophagy inhibitors due to their action on suppression of lysosome formation and its function. ${ }^{82}$ Chloroquine has been used in combination with many chemotherapeutic agents and a large number of trials are ongoing on its effect on cancer therapy alone or in various combinations. ${ }^{83}$

\section{Targeting Autophagy in Acute Liver Injury}

In acute liver injury, autophagy has a protective role and autophagic cell death occurs in failure of adaptation. Cell death in acute liver injury is mediated by TNF in many cases. A recent study has demonstrated that pharmacological induction of autophagy in an in-vivo model mediates cellular resistance to TNF toxicity by blocking activation of caspase- 8 and thus preventing mitochondrial death. ${ }^{84}$

In experimental models of acetaminophen induced liver injury, induction of autophagy by Rapamycin was found to be protective and suppression of autophagy by chloroquine exacerbated liver injury. In this model, autophagy was found to be acting mainly by removing the acetaminophen damaged mitochondria.

\section{Targeting Autophagy in Liver Ischemia Reperfusion and Liver Surgery}

Liver ischemia and reperfusion injury occurs in many conditions like shock, liver transplantation, liver resection and liver trauma. Cellular damage due to hypoxia during period of low blood flow is further accentuated by inflammatory mediators due to return of blood flow after reperfusion. Various mechanisms are being studied to ameliorate the liver injury. Autophagy has been studied in this context using mouse models with ischemia induced by portal triad occlusion followed by reperfusion or no reperfusion. The results in various studies have been

Table 1 Role of Autophagy in Various Liver Diseases and the Agents Targeting Autophagy.

\begin{tabular}{|c|c|c|c|c|}
\hline Liver disease & $\begin{array}{c}\text { Hepatic autophagy } \\
\text { level }\end{array}$ & Role of autophagy & $\begin{array}{l}\text { Autophagy } \\
\text { modulation }\end{array}$ & $\begin{array}{l}\text { Agents used } \\
\text { to target autophagy }\end{array}$ \\
\hline $\begin{array}{l}\text { Fatty liver } \\
\text { disease }\end{array}$ & Decreased & Lipid degradation, insulin signaling & Autophagy inducers & TORIN-1, Rapamycin \\
\hline $\begin{array}{l}\text { Alcoholic liver } \\
\text { disease }\end{array}$ & Decreased & $\begin{array}{l}\text { Degradation of protein aggregates } \\
\text { and damaged organelles }\end{array}$ & Autophagy inducers & TORIN-1, Rapamycin \\
\hline \multirow[t]{2}{*}{$\begin{array}{l}\text { Hepatocellular } \\
\text { carcinoma }\end{array}$} & Decreased & $\begin{array}{l}\text { Anti-tumor effect } \\
\text { in normal cells; } \\
\text { Autophagy is used by cancer } \\
\text { cells for survival against } \\
\text { apoptosis by traditional } \\
\text { chemotherapeutic drugs }\end{array}$ & Autophagy inducers & $\begin{array}{l}\text { Rapamycin, Everolimus, } \\
\text { Bortezomib, } \\
\text { Sorafenib, NPC-16, }\end{array}$ \\
\hline & & & Autophagy inhibitors & $\begin{array}{l}\text { 3MA, Chloroquine, } \\
\text { SiRNA, MicroRNAs }\end{array}$ \\
\hline \multirow[t]{2}{*}{ HBV Infection } & Increased & Autophagy subverted by virus & Autophagy suppressor & 3MA \\
\hline & & & Autophagy inducer & Rapamycin \\
\hline \multirow[t]{2}{*}{ HCV infection } & Increased & Autophagy subverted by virus & Autophagy suppressor & 3MA, Chloroquine \\
\hline & & & Autophagy inducer & Rapamycin \\
\hline A1-AT deficiency & - & $\begin{array}{l}\text { Autophagy clears the AT } \\
\text { protein aggregation }\end{array}$ & $\begin{array}{l}\text { mTOR independent } \\
\text { increase in autophagy }\end{array}$ & Carbamazepine \\
\hline
\end{tabular}


contradictory. ${ }^{86,87}$ However, in studies of liver transplantation with cold ischemia time of $24 \mathrm{~h}$ followed by reperfusion, autophagy induction has been found to decrease liver cell death. ${ }^{8}$

\section{Targeting Autophagy in Drug Induced Liver Disease}

Since many drugs are metabolized in the liver, the liver is consequently a major target of damage due to drugs. Drug induced liver injury may result in drug discontinuation and has the potential of acute liver failure. Acetaminophen toxicity is a major cause of liver injury and can lead to liver failure and death. Liver injury is mediated by mitochondrial dysfunction. In experimental animal models, induction of autophagy has been shown to play a role in protecting the liver against drug induced hepatotoxicity by mitophagy and reducing oxidative stress. ${ }^{89}$ Pharmacological modification of autophagy may provide a novel therapeutic approach in drug induced liver injury.

\section{CONCLUSION}

The primary role of autophagy is in cell survival. However, in certain conditions it can lead to autophagic cell death. Viral persistence and multiplication in Hepatitis B and C infection is mediated by the subversion of autophagy. With the elucidation of the role of autophagy in multiple facets of liver diseases, its modulation is likely to emerge as a potential therapeutic target in emerging therapies. The role of autophagy in various liver diseases and the agents targeting autophagy are depicted in Table 1. However, much work needs to be done in understanding the exact role of autophagy in diseases like HCC, measurement of state of autophagy and development of drugs for modulating autophagy before novel therapies targeting autophagy become a reality in the near future.

\section{CONFLICTS OF INTEREST}

All authors have none to declare.

\section{REFERENCES}

1. Hruban Z, Spargo B, Swift H, Wissler RW, Kleinfeld RG. Focal cytoplasmic degradation. Am J Pathol. 1963;42:657-683.

2. Arstila AU, Trump BF. Studies on cellular autophagocytosis. The formation of autophagic vacuoles in the liver after glucagon administration. Am J Pathol. 1968;53:687-733.

3. Klionsky DJ, Cregg JM, Dunn Jr WA, et al. A unified nomenclature for yeast autophagy-related genes. Dev Cell. 2003;5:539-545.

4. Rubeinsztein DC, Martino G, Kroemer G. Autophagy and aging. Cell. 2011;146:682-695.

5. Laplante M, Sabatini DM. mTOR signaling in growth control and disease. Cell. 2012;13:274-293.

6. Ravikumar B, Vacher C, Berger Z, et al. Inhibition of mTOR induces autophagy and reduces toxicity of polyglutamine expansions in fly and mouse models of Huntington disease. Nat Genet. 2004;36: 585-595.
7. Agarraberes FA, Terlecky SR, Dice JF. An intralysosomal hsp70 is required for a selective pathway of lysosomal protein degradation. J Cell Biol. 1997;137:825-834.

8. Terlecky SR, Dice JF. Polypeptide import and degradation by isolated lysosomes. J Biol Chem. 1993;268:23490-23495.

9. Nakatogawa H, Suzuki K, Kamada Y, Ohsumi Y. Dynamics and diversity in autophagy mechanisms: lessons from yeast. Nat Rev Mol Cell Biol. 2009;10:458-467.

10. Xie Z, Klionsky DJ. Autophagosome formation: core machinery and adaptations. Nat Cell Biol. 2007;9:1102-1109.

11. Yang Z, Klionsky DJ. Mammalian autophagy: core molecular machinery and signaling. Curr Opin Cell Biol. 2010;22(2):124-131.

12. Hosokawa N, Hara T, Kaizuka T, et al. Nutrient-dependent mTORC1 association with the ULK1-Atg13-FIP200 complex required for autophagy. Mol Biol Cell. 2009;20:1981-1991.

13. Yang Z, Klionsky DJ. An overview of the molecular mechanism of autophagy. Curr Top Microbiol Immunol. 2009;335:1-32.

14. Fujita $\mathrm{N}$, Itoh $\mathrm{T}$, Omori $\mathrm{H}$, Fukuda M, Noda T, Yoshimori T. The Atg16L complex specifies the site of LC3 lipidation for membrane biogenesis in autophagy. Mol Biol Cell. 2008;19:2092-2100.

15. Zhong Y, Wang QJ, Li X, et al. Distinct regulation of autophagic activity by Atg14L and Rubicon associated with Beclin 1-phosphatidylinositol3-kinase complex. Nat Cell Biol. 2009;11:468-476.

16. Liang C, Lee JS, Inn KS, et al. Beclin1-binding UVRAG targets the class C Vps complex to coordinate autophagosome maturation and endocytic trafficking. Nat Cell Biol. 2008;10:776-787.

17. Reggiori F, Tucker KA, Stromhaug PE, Klionsky DJ. The Atg1-Atg13 complex regulates Atg9 and Atg23 retrieval transport from the preautophagosomal structure. Dev Cell. 2004;6:79-90.

18. Calvo-Garrido J, Carilla-Latorre S, Lazaro-Dieguez F, Egea G, Escalante R. Vacuole membrane protein 1 is an endoplasmic reticulum protein required for organelle biogenesis, protein secretion and development. Mol Biol Cell. 2008;19:3442-3453.

19. Chen Y, Klionsky DJ. The regulation of autophagy - unanswered questions. J Cell Sci. 2011;124:161-170.

20. Komatsu M. Liver autophagy: physiology and pathology. J Biochem. 2012;152(1):5-15.

21. Fimia GM, Piacentini M. Regulation of autophagy in mammals and its interplay with apoptosis. Cell Mol Life Sci. 2010;67: 1581-1588.

22. Hou W, Han J, Lu C, Goldstein LA, Rabinowich H. Autophagic degradation of active caspase-8: a crosstalk mechanism between autophagy and apoptosis. Autophagy. 2010;6:891-900.

23. Maiuri C, Zalckvar E, Kimchi A, Kroemer G. Self-eating and selfkilling: crosstalk between autophagy and apoptosis. Nat Rev Mol Cell Biol. 2007;8:741-752.

24. Mortimore GE, Hutson NJ, Surmacz CA. Quantitative correlation between proteolysis and macro- and microautophagy in mouse hepatocytes during starvation and refeeding. Proc Natl Acad Sci U S A. 1983;80:2179-2183.

25. Ezaki J, Matsumoto N, Takeda-Ezaki M, et al. Liver autophagy contributes to the maintenance of blood glucose and amino acid levels. Autophagy. 2011;7:727-736.

26. Singh R, Kaushik S, Wang Y, et al. Autophagy regulates lipid metabolism. Nature. 2009;458:1131-1135.

27. Mizushima N, Komatsu M. Autophagy: renovation of cells and tissues. Cell. 2011;147:728-741.

28. Komatsu M, Waguri S, Ueno T, et al. Impairment of starvationinduced and constitutive autophagy in Atg7-deficient mice. J Cell Biol. 2005;169:425-434.

29. Hidvegi T, Ewing M, Hale P, et al. An autophagy-enhancing drug promotes degradation of mutant alpha1-antitrypsin Z and reduces hepatic fibrosis. Science. 2010;329:229-232.

30. Mathew R, Karantza-Wadsworth V, White E. Role of autophagy in cancer. Nat Rev Cancer. 2007;7:961-967. 
31. Levine B, Kroemer G. Autophagy in the pathogenesis of disease. Cell. 2008;132:27-42.

32. Feng $\mathrm{Z}$, Zhang $\mathrm{H}$, Levine $\mathrm{AJ}$, Jin $\mathrm{S}$. The coordinate regulation of the p53 and mTOR pathways in cells. Proc Natl Acad Sci U S A. 2005;102:8204-8209.

33. Menon S, Yecies JL, Zhang HH, et al. Chronic activation of mTOR complex 1 is sufficient to cause hepatocellular carcinoma in mice. Sci Signal. 2012;5:ra24.

34. Crighton D, Wilkinson S, O'Prey J, et al. DRAM, a p53-induced modulator of autophagy, is critical for apoptosis. Cell. 2006;126: 121-134.

35. Tasdemir E, Maiuri MC, Galluzzi L, et al. Regulation of autophagy by cytoplasmic p53. Nat Cell Biol. 2008;10:676-687.

36. Meléndez A, Tallóczy Z, Seaman M, Eskelinen EL, Hall DH, Levine B. Autophagy genes are essential for dauer development and life-span extension in C. elegans. Science. 2003;301:1387-1391.

37. Lipinski MM, Zheng B, Lu T, et al. Genome-wide analysis reveals mechanisms modulating autophagy in normal brain aging and in Alzheimer's disease. Proc Natl Acad Sci U S A. 2010;107: 14164-14169.

38. Caramés B, Taniguchi N, Otsuki S, Blanco FJ, Lotz M. Autophagy is a protective mechanism in normal cartilage, and its aging-related loss is linked with cell death and osteoarthritis. Arthritis Rheum. 2010;62:791-801.

39. Decuypere JP, Monaco G, Missiaen L, De Smedt H, Parys JB, Bultynck G. IP(3) receptors, mitochondria, and Ca signaling: implications for aging. J Aging Res. 2011:920178.

40. Colman RJ, Anderson RM, Johnson SC, et al. Caloric restriction delays disease onset and mortality in rhesus monkeys. Science. 2009;325:201-204.

41. Pattingre S, Tassa A, Qu X, et al. Bcl-2 antiapoptotic proteins inhibit Beclin 1-dependent autophagy. Cell. 2005;122:927-939.

42. Maiuri MC, Tasdemir E, Criollo A, et al. Control of autophagy by oncogenes and tumor suppressor genes. Cell Death Differ. 2009;16:87-93.

43. Mathew R, Karp CM, Beaudoin B, et al. Autophagy suppresses tumorigenesis through elimination of p62. Cell. 2009;137:1062-1075.

44. Levine B, Mizushima N, Virgin HW. Autophagy in immunity and inflammation. Nature. 2011;469:323-335.

45. Zhang C, Cuervo AM. Restoration of chaperone-mediated autophagy in aging liver improves cellular maintenance and hepatic function. Nat Med. 2008;14:959-965.

46. Kim I, Rodriguez-Enriquez S, Lemasters JJ. Selective degradation of mitochondria by mitophagy. Arch Biochem Biophys. 2007;462: 245-253.

47. Mortimore GE, Poso AR. Intracellular protein catabolism and its control during nutrient deprivation and supply. Annu Rev Nutr. 1987;7:539-564.

48. Meijer AJ, Codogno P. Regulation and role of autophagy in mammalian cells. Int J Biochem Cell Biol. 2004;36:2445-2462.

49. Mihaylova MM, Shaw RJ. The AMPK signalling pathway coordinates cell growth, autophagy and metabolism. Nat Cell Biol. 2011;13: 1016-1023.

50. Kotoulas OB, Kalamidas SA, Kondomerkos DJ. Glycogen autophagy. Microsc Res Tech. 2004;64:10-20.

51. Ni HM, Williams JA, Yang H, Shi YH, Fan J, Ding WX. Targeting autophagy for the treatment of liver diseases. Pharm Res. 2012;66: 463-474.

52. Czaja MJ. Functions of autophagy in hepatic and pancreatic physiology and disease. Gastroenterology. 2011;140:1895-1908.

53. Yang L, Li P, Fu S, Calay ES, Hotamisligil GS. Defective hepatic autophagy in obesity promotes ER stress and causes insulin resistance. Cell Metab. 2010;11:467-478.

54. Liu HY, Han J, Cao SY, et al. Hepatic autophagy is suppressed in the presence of insulin resistance and hyperinsulinemia: inhibition of FOX01-dependent expression of key autophagy genes by insulin. J Biol Chem. 2009;284(45):31484-31492.

55. Gozuacik D, Kimchi A. Autophagy and cell death. Curr Top Dev Biol. 2007;78:217-245.

56. Caldwell SH, Swerdlow RH, Khan EM, et al. Mitochondrial abnormalities in non-alcoholic steatohepatitis. J Hepatol. 1999;31(3): 430-434.

57. Singh $R$, Xiang $Y$, Wang $Y$, et al. Autophagy regulates adipose mass and differentiation in mice. J Clin Invest. 2009;119(11): 3329-3339.

58. Peterson TR, Sengupta SS, Harris TE, et al. mTOR complex 1 regulates lipin 1 localization to control the SREBP pathway. Cell. 2011;146:408-420.

59. Amir M, Czaja MJ. Autophagy in nonalcoholic steatohepatitis. Expert Rev Gastroenterol Hepatol. 2011;5(2):159-166.

60. Donohue Jr TM, Zetterman RK, Tuma DJ. Effect of chronic ethanol administration on protein catabolism in rat liver. Alcohol Clin Exp Res. 1989;13:49-57.

61. Harada M, Hanada S, Toivola DM, Ghori N, Omary MB. Autophagy activation by rapamycin eliminates mouse Mallory-Denk bodies and blocks their proteasome inhibitor-mediated formation. Hepatology. 2008;47:2026-2035.

62. Donohue Jr TM, McVicker DL, Kharbanda KK, Chaisson ML, Zetterman RK. Ethanol administration alters the proteolytic activity of hepatic lysosomes. Alcohol Clin Exp Res. 1994;18: 536-541.

63. Routou PE, Mansouri A, Lebrec D, Durand F, Valla D, Moreau R. Autophagy in liver diseases. J Hepatol. 2010;53:1123-1134.

64. Perlmutter DH. Autophagic disposal of the aggregation-prone protein that causes liver inflammation and carcinogenesis in alpha1-antitrypsin deficiency. Cell Death Differ. 2009;16:39-45.

65. Puls F, Goldschmidt I, Bantel H, et al. Autophagy-enhancing drug carbamazepine diminishes hepatocellular death in fibrinogen storage disease. J Hepatol. 2013;59:626-630.

66. Kudchodkar SB, Levine B. Viruses and autophagy. Rev Med Virol. 2009;19:359-378.

67. Dreux M, Gastaminza P, Wieland SF, Chisari FV. The autophagy machinery is required to initiate hepatitis $\mathrm{C}$ virus replication. Proc Nat Acad Sci U S A. 2009;106:14046-14051.

68. Sir D, Chen WL, Choi J, Wakita T, Yen TS, Ou JH. Induction of incomplete autophagic response by hepatitis $\mathrm{C}$ virus via the unfolded protein response. Hepatology. 2008;48:1054-1061.

69. Rautou PE, Cazals-Hatem D, Feldmann G, et al. In vivo evidence of altered hepatocyte autophagic response in livers from patients with chronic Hepatitis C virus infection. Hepatology. 2009;50:943A.

70. Ait-Goughoulte M, Kanda T, Meyer K, Ryerse JS, Ray RB, Ray R. Hepatitis $C$ virus genotype 1 a growth and induction of autophagy. J Virol. 2008;82:2241-2249.

71. Sir D, Tian Y, Chen WL, Ann DK, Yen TS, Ou JH. The early autophagic pathway is activated by hepatitis $B$ virus and required for viral DNA replication. Proc Natl Acad Sci U S A. 2010;107:4383-4388.

72. Tang $H$, Da L, Mao $Y$, et al. Hepatitis $B$ virus $X$ protein sensitizes cells to starvation-induced autophagy via up-regulation of beclin1 expression. Hepatology. 2009;49:60-71.

73. Thoen LF, Guimaraes EL, Dolle L, et al. A role for autophagy during hepatic stellate cell activation. J Hepatol. 2011;55:1353-1360.

74. Qu X, Yu J, Bhagat G, et al. Promotion of tumorigenesis by heterozygous disruption of the beclin 1 autophagy gene. J Clin Invest. 2003;112:1809-1820.

75. Ding ZB, Shi YH, Zhou J, et al. Association of autophagy defect with a malignant phenotype and poor prognosis of hepatocellular carcinoma. Cancer Res. 2008;68:9167-9175.

76. Cui J, Gong Z, Shen HM. The role of autophagy in liver cancer: molecular mechanisms and potential therapeutic targets. Biochim Biophys Acta. 2013;1836:15-26. 
77. Peng YF, Shi YH, Shen YH, et al. Promoting colonization in metastatic HCC cells by modulation of autophagy. PLoS One. 2013;8(9): e74407.

78. Amaravadi RK, Lippincott-Schwartz J, Yin XM, et al. Principles and current strategies for targeting autophagy for cancer treatment. Clin Cancer Res. 2011;17:654-666.

79. Huynh H, Pierce Chow K, Soo KC, et al. RAD001 (everolimus) inhibits tumour growth in xenograft models of human hepatocellular carcinoma. J Cell Mol Med. 2009;13:1371-1380.

80. Arora A, Scholar EM. Role of tyrosine kinase inhibitors in cancer therapy. J Pharmacol Exp Ther. 2005;315:971-979.

81. Park MA, Zhang G, Martin AP, et al. Vorinostat and sorafenib increase ER stress, autophagy and apoptosis via ceramide-dependent CD95 and PERK activation. Cancer Biol Ther. 2008;7:1648.

82. Poole B, Ohkuma S. Effect of weak bases on the intralysosomal pH in mouse peritoneal macrophages. J Cell Biol. 1981;90:665-669.

83. Shimizu S, Takehara T, Hikita H, et al. Inhibition of autophagy potentiates the antitumor effect of the multikinase inhibitor sorafenib in hepatocellular carcinoma. Int J Cancer. 2012;131:548-557.
84. Amir M, Zhao E, Fontana L, et al. Inhibition of hepatocyte autophagy increases tumor necrosis factor-dependent liver injury by promoting caspase-8 activation. Cell Death Differ. 2013;20:878-887.

85. Ni HM, Jaeschke H, Ding WX. Targeting autophagy for drug induced hepatotoxicity. Autophagy. 2012;8:709-710.

86. Kim JS, Nitta T, Mohuczy D, et al. Impaired autophagy: a mechanism of mitochondrial dysfunction in anoxic rat hepatocytes. Hepatology. 2008;47:1725-1736.

87. Igusa Y, Yamashina S, Izumi K, et al. Loss of autophagy promotes murine acetaminophen hepatotoxicity. J Gastroenterol. 2012; 47(4):433-443.

88. Cardinal J, Pan P, Dhupar R, et al. Cisplatin prevents high mobility group box 1 release and is protective in a murine model of hepatic ischemia/reperfusion injury. Hepatology. 2009;50: $565-574$.

89. Domart MC, Esposti DD, Sebagh M, et al. Concurrent induction of necrosis, apoptosis, and autophagy in ischemic preconditioned human livers formerly treated by chemotherapy. J Hepatol. 2009;51:881-889. 